\title{
Refinement and Experimental Verification of a Model for Fire Growth and Smoke Transport
}

\author{
WALTER W. JONES and RICHARD D. PEACOCK \\ Center for Fire Research \\ National Bureau of Standards \\ Gaithersburg, Maryland 20899, USA
}

\begin{abstract}
There is considerable interest in modeling the growth of fires and the spread of toxic gases in multicompartment structures. Much of the attention is focused on the development of numerical models which are fast and robust, but able to make reasonably accurate predictions from the onset of ignition. We have constructed such a model (FAST) and performed a series of validation experiments to test it. This paper is a discussion of some of the improvements which have been made to physical algorithms and underlying numerical basis of the model, a description of some of the experiments used to verify the refined model, and of some additions which we intend to incorporate.
\end{abstract}

\section{INTRODUCTION}

Considerable research has been done regarding the spread of fire and smoke from a room of fire origin to connected compartments. The work is motivated by a need to understand and predict the environmental conditions which occur as a fire develops and spreads. Much of the attention has focused on the development of numerical models which are able to make a reasonably accurate assessment of the environment from ignition. We have built such a mode1, FAST. The basis of the model has been discussed elsewhere [1]. As a result of extensive validation work which has been done with the model, we have made several refinements. We start with a very brief description of the predictive equations which are contained in the original model. This is done to provide a basis for discussion. The refinements are then discussed in terms of the original formulation of the source terms for these predictive equations. Finally, we show a sample of the experimental results which were used to verify that the model has indeed improved.

\section{STRUCTURE OF THE MODEL}

The primary element of our model is the compartment. Predictive equations for the gas layers in each compartment are derived from conservation of mass, momentum and energy, an equation of state and the boundary conditions to which each compartment is subject. The term "boundary condition" refers to the transfer points at the boundaries of the compartments; examples are vents, air conditioning ducts, etc. The actual physical phenomena which drive the transport are then couched as source terms. Such a formulation allows the greatest flexibility in 
adding, modifying, or deleting terms which are appropriate to the problem at hand.

Each compartment is subdivided into "control volumes." The premise is that the details which occur within such a volume do not concern us (at present), but their interaction does. Each control volume is called a zone. Such a choice arises from the observation that when a fire grows and spreads, the gas layers in a compartment stratify into distinct zones. In the present calculation we use only two zones per compartment. The choice of two is based on the observation that there is reasonably good agreement between theory and experiment for this choice, and there are other phenomena which put a more severe constraint on the validity of the model. An example of a compartment which might reasonably contain more than two zones would be a long corridor whose aspect is greater than 10.

The general layout of the zones and the form of the conservation equations is discussed elsewhere[1]. The focus of this presentation is the explicit form of the pressure equation and reference point for this variable. The hydrostatic term is not significant when compared to the absolute pressure of a compartment nor is it large in comparison with typical pressure fluctuations. It is important in calculating pressure differences across openings between compartments. So in the spirit of the "control volume" approach we assume that the pressure is a constant within the zone when considering the conservation equations. The reference point for this pressure is at the base of the compartment. The associated simplification is that we solve the momentum equation in an integral form as a boundary condition for the zone, that is, Bernoul11's equation is used to calculate the flow between compartments. It includes the hydrostatic term even though it is not included in the conservation equations explicitly. A stratified medium will support both acoustic and gravity waves. They do not materially influence the phenomena of interest at present, but do put a constraint on the time step allowed. Our stratagem eliminates this type of wave motion, at least for individual compartments, and thereby allows a much larger time step.

\section{PREDICTIVE EQUATIONS}

A zone model describes a physical situation in terms of integrals of physical quantities. For example, total mass is calculated for a compartment rather than mass density and total energy rather than energy density. The integrals are volume integrals whose boundary surfaces enclose the Euclidean space of interest. Usually we assume that the intensive variables are constant within a zone. This is not a necessary restriction but is done for ease of derivation and speed of computation. The space with which we are concerned usually consists of several compartments with a hot upper zone and a relatively cool lower zone for each compartment, together with objects such as chairs, plumes and fires. Interactions between zones occur at the boundaries of the zones. Examples of possible interactions are the flow through the vents connecting compartments, the radiation from one compartment through a vent to another compartment and a plume which connects the upper zone and the lower zone of a compartment.

The predictive equation for the pressure is

$\frac{\mathrm{dP}}{\mathrm{dT}}=\frac{\dot{\mathrm{s}}}{(\beta-1) \mathrm{V}}$ 
where $\dot{\mathrm{s}}=\mathrm{c}_{\mathrm{p}} \dot{\mathrm{m}}_{\mathrm{u}} \mathrm{T}_{\mathrm{u}}+\mathrm{c}_{\mathrm{p}} \dot{\mathrm{m}}_{\ell} \mathrm{T}_{\ell}+\dot{\mathrm{E}}_{\mathrm{u}}+\dot{\mathrm{E}}_{\ell}$ and $\beta=\mathrm{c}_{\mathrm{p}} / \mathrm{R}=\gamma /(\gamma-1)$. This equation is applied to each compartment.

The form of the energy terms $\left(\dot{E}_{u}, \dot{E}_{\ell}\right)$ is important. The form given in reference [1] is physically correct but does not work very well because of the large effect a slight change in $\mathrm{p}$ has on $\dot{m}$. In particular, when a fire is at steady state or decreasing, the layer temperatures should equilibrate or decrease to ambient respectively. In the earlier work, the reference temperature was set to zero. This choice is used in all other zone model derivations. A better choice turns out to be the ambient. An alternative to recasting the equations is to use double precision numerics for the computation. Such a choice increases the computing time and storage requirements by as much as a factor of two. By recasting all enthalpy flux terms as differences of mass flow in and out, and temperature minus the reference ambient, it is not necessary to invoke double precision, since these changes reduce the required precision by two orders of magnitude. The primary effect of such a choice is that the pressure term is now sensitive to pressure differences of $\leq 0.1$ Pascals. With the choice $\mathrm{T}_{\mathrm{R}} \rightarrow \mathrm{T}_{\mathrm{a}}$, we have

$\dot{\mathrm{E}}_{\mathrm{j}}=\dot{\mathrm{Q}}_{\mathrm{f}}(j)+\dot{\mathrm{Q}}_{\mathrm{R}}(j)+\dot{\mathrm{Q}}_{\mathrm{c}}(j)$

$$
\begin{aligned}
& +\sum_{i} c_{p} \dot{m}_{i, j}\left(T_{i}-T_{u}\right)+R\left(T_{a}-T_{u}\right) \dot{m}_{i, j} \\
& +\sum_{i} c_{p} \dot{m}_{i, j}\left(T_{j}-T_{k}\right)
\end{aligned}
$$

and the source term "s" becomes

$$
\begin{aligned}
\dot{\mathrm{s}}=\sum_{j} \dot{\mathrm{Q}}_{\mathrm{f}}(j) & +\dot{\mathrm{Q}}_{\mathrm{R}}(j)+\dot{\mathrm{Q}}_{c}(j) \\
& +\sum_{j} \sum_{\dot{j}} c_{p} \dot{\mathrm{m}}_{i, j} \operatorname{in}_{j}\left(\mathrm{~T}_{i}-\mathrm{T}_{j}\right)+c_{\mathrm{p}} \dot{\mathrm{m}}_{j}\left(\mathrm{~T}_{j}-\mathrm{T}_{\mathrm{a}}\right)+c_{\mathrm{v}} \dot{\mathrm{m}}_{j} \mathrm{~T}_{\mathrm{a}}
\end{aligned}
$$

where

$$
\begin{aligned}
& \dot{m}_{i, j}=\dot{m}_{i, j} i_{j}-\dot{m}_{i, j}, u t \\
& \dot{m}_{j}=\sum_{i} \dot{m}_{i, j}^{i n}-\dot{m}_{i}{ }_{i, j} u_{j} t \\
& \mathrm{~T}_{\mathrm{k}}= \begin{cases}\mathrm{T}_{\mathrm{u}} & \text { if } j=" \ell " \\
\mathrm{~T}_{\ell} & \text { if } j=" \mathrm{u}^{\prime} .\end{cases}
\end{aligned}
$$

The index " $j$ " is for the layers " $u$ " and " $\ell$ " and " $i$ " is for the compartments which have connections to the compartment under consideration. If there is more than one connection between the compartments, then this 
latter summation is multivalued. In addition, for each compartment in which a plume is present there is a term for the reduction in the energy release for bringing the fuel and entrained air from its initial temperature to that of the upper layer.

\section{SOURCE TERMS}

The predictive equations are arranged so that the physical phenomena are source terms on the right-hand-side of these equations. Such a formulation makes the addition (and deletion) of physical phenomena and changing the form of algorithms a relatively simple matter. For the most part, the source terms are as discussed in ref[1]. The exception to this is the method of dealing with flow through vents.

Flow at vents is governed by the pressure difference across a vent. In the control volume approximation, the differential form of the momentum equation for the zones is not solved. Instead the momentum txansfer at the zone boundaries is included by using Bernoulli's solution for the velocity equation. This solution is augmented for restricted openings by using flow coefficients[2] to allow for constriction in actual vents. The modifications deal with the problem of constriction of velocity streamlines at an orifice.

Flow is the dominant term in solving the conservation equations because it fluctuates the most rapidly of all the source terms and in turn is most sensitive to changes in the environment. One of the improvements which we have incorporated into our current models is a means of calculating these flow fields with the correct number of neutral planes[3]. It is possible to have up to three neutral planes. When discussing the interaction between two compartments, there is two-fold symmetry, so we only need to consider one of the two cases. Any criterion can be used to break the symmetry; we use the ordering of the upper layer densities, $\rho_{1}<\rho_{3}$.

The general form for flow is given by

$\dot{\mathrm{m}}_{i 0}=\mathrm{CS} \sqrt{2 \rho\left|\mathrm{P}_{i}-\mathrm{P}_{0}\right|}$

where $\dot{m}$ is the mass flow rate, $C$ is an orifice coefficient $(\approx 0.7), S$ is the opening area, $p$ is the gas density on the source side and $P$ is the pressure on the source(i) and destination(o) sides respectively.

The implication of using this equation is that the pressure at a stagnation point is used. That is, the flow velocity vanishes where the pressure is measured. We apply the above equation to rectangular openings which allows us to remove the width from the mass flux integral. That is

flow $=\int_{\text {width }} \int_{\text {height }} \rho \mathrm{V} d z d b+$ width $\int_{z_{1}}^{z_{2}} \rho \mathrm{V} d z$

The simplest means to define the limits of integration is with neutral planes or physical boundaries such as a sill or soffit. By breaking the integral into intervals defined by flow reversal, a soffit, a sill, or a zone interface, the integral itself can be done analytically. We have for the internal pressure on each side of the opening 
$P_{i}(z)=P_{i}(0)-\min \left(z, Z_{i}\right) \rho_{2} g-\max \left(z-Z_{i}, 0\right) \rho_{1} g$

$P_{0}(z)=P_{0}(0)-\min \left(z, Z_{0}\right) \rho_{4} g-\max \left(z-Z_{0}, 0\right) \rho_{3} g$

where $P(0)$ represents the base (reference) pressure at the floor. The pressure then appears only as a difference of these two terms, namely $F(z)=P_{i}(z)-P_{0}(z)$. These equations form an inordinately large family of curves as a function of the parameters $\rho$ and $Z$. However, if the restrictions found in fire scenarios are imposed then we end up with only a few possibilities.

If there were no soffits or sills to consider, then the calculation would be fairly straightforward. However, the possibility of soffit/sill combinations requires many numerical tests in the calculation. For the case of one neutral plane or fewer, there are 44 different flow combinations, depending on the relative position of sills, soffits and interface positions. Twenty-four of these combinations are without a neutral plane and twenty with a neutral plane. For all cases of more than one neutral plane, each interval can be partitioned into intervals which contain at most a single neutral plane and the logic used for this evaluation can be utilized.

When one of the limits of integration is $F(z)=0$, that is a neutral plane, the mass flow over an interval $\left(z_{2}-z_{1}\right)$ is given by

$\dot{\mathrm{m}}_{\mathrm{i} \rightarrow 0}=\frac{2}{3} \mathrm{CS} \sqrt{2 \rho\left|\mathrm{P}_{1}-\mathrm{P}_{0}\right|}+\frac{2}{3} \mathrm{CS}\left(\mathrm{z}_{2}-\mathrm{z}_{1}\right) \sqrt{2 \rho\left|\mathrm{P}_{i}\left(z_{2}\right)-\mathrm{P}_{0}\left(z_{2}\right)\right|}$

and for the case of no neutral plane, we obtain

$\dot{\mathrm{m}}_{i+0}=\frac{2}{3} \operatorname{CS} \sqrt{2 \rho\left(z_{2}-z_{1}\right)} \frac{1}{\left(\mathrm{P}_{2}-\mathrm{P}_{1}\right)}\left(\mathrm{P}_{2}^{3 / 2}-\mathrm{P}_{1}^{3 / 2}\right)$

where $\rho$ is the average inlet mass density within the area. The flow will be in the opposite direction if $P_{0}>P_{1}$. The pressure at $z_{1}$ is $P_{1}$ and at $z_{2}$ is $p_{2}$. For the purposes of illustration, we have assumed the pressure difference at $z_{1}$ to be zero for the case of a neutral plane. The integration is started at the lowest point at which flow can occur, the sill or floor. Then the next change point is calculated. It is either a soffit or a change in the relative gas density. Within this interval there is either a neutral plane or not. In either case, the flow equation can be integrated analytically. In the former case, the bidirectional flow is calculated from the neutral plane to the two end points. The evaluation of this function is quite fast. In the latter case the solution can better be expressed as a continued fraction. The form becomes

$\mathrm{x} \equiv \operatorname{minimum} \mathrm{P}_{\frac{1}{2}} / 2, \mathrm{P}_{1}^{1} / 2$

$y=\operatorname{maximum} \mathrm{P}_{2}^{1 / 2}, \mathrm{P}_{1}^{1 / 2}$

$\dot{\mathrm{m}}_{\mathrm{i}+0}=\frac{2}{3} \mathrm{CS} \sqrt{2 \rho}\left(\mathrm{z}_{2}-\mathrm{z}_{1}\right) \frac{\mathrm{x}+\mathrm{y}}{1+\mathrm{x} / \mathrm{y}}$ 
with a maximum error of $5 \%$ for $x / y=0.5$, and this latter calculation is considerably faster. A check is then made to see if there is more opening through which flow can occur. If so, then the integration process starts from the last endpoint $\left(z_{2}\right)$ and continues until the soffit is reached.

Currently only a specified fire is implemented. It can be run in the unconstrained, or constrained mode. In the unconstrained mode, the fire will release energy as specified, For this case, the major species are not tracked. Calculations can be made for optical depth and toxicity, however. In the constrained mode, a full set of species calculations are done, including transport of nitrogen, depletion of oxygen, production and depletion of unburned fuel, and production of $\mathrm{CO}_{2}, \mathrm{CO}$, $\mathrm{H}_{2} \mathrm{O}$, $\mathrm{HCN}$ and $\mathrm{HCL}$. The burning rate is then limited to the oxygen available, either in the plume, or during entrainment at vents.

The form of the species equation is similar for all species and all species are treated the same in the model. There are some differences in detail, however. For example, HCL has a surface deposition term which is not present for $\mathrm{CO}_{2}$.

At present the model considers four boundary surfaces for each compartment. These are labeled ceiling, upper wall, lower wall and floor. The thermophysical properties of the upper and lower wall are assumed to be the same, but can differ from the floor and ceiling. Conduction is done on a compartment by compartment basis, with each bounding surface specified and calculated independently. Any combination of compartments and surfaces within a compartment can be specified. At present conduction is one dimensional only, perpendicular to the bounding surface. This is a limitation in moving from compartments near the fire source to distant spaces. An important mechanism for complete mixing is flow down the walls and the degree of mixing is affected by heat conduction parallel to the wall, especially in the direction in which the interface is moving. Goldman et al. [4] have shown that the difference in the thermoclines of the wall and gas layer can be important, since it will influence the direction in which the wall boundary flow propagates.

\section{EXPERIMENTAL VERIFICATION}

Several full scale experiments have been performed for testing this model. The experimental arrangement consisted of a burn room and a $12.19 \mathrm{~m}$ long corridor with a target room on one side. A diffusion flame burner using natural gas served as the fire source.

In an attempt to develop statistically meaningful data for each test situation, each test combination of fire size and corridor configuration was repeated numerous times. The primary data from the experiments was provided by thermocouples, smoke meters and pressure probes. Details of the methodology used in the experiment are discussed elsewhere[5].

\section{COMPARISON BETWEEN EXPERIMENTAL DATA AND THEORETICAL PREDICTIONS}

The measured quantities for which we will make a direct comparison between an experiment and the corresponding theoretical prediction are the upper layer temperature, the interface height and mass flow into (or out) of a compartment. There are other measurements of interest, but these four will yield an indication of the match of the model to the 
experiment. The temperature of the lower layer is important when considering the effect on occupants and equipment, but there can not yet be a good correspondence due to the assumption of no radiative heating. The absence of lower layer heating will be discussed in the context of agreement with other data.

Figure 1 shows the upper layer temperature as a function of time for the three rooms in the test facility. Figure 2 shows similar information for the interface height. The agreement for many of the variables is quite good, but systematic deviations do exist. Convection is the mechanism for enthalpy flux between layers and compartments. In the burn room, the plume dominates and in adjacent compartments the flow through vents is most important. In the earlier version of this model, the plume was not treated as a separate zone. Rather we assumed that the upper layer was connected to the fire by the plume. The implication is that the plume is formed instantaneously. Consequently, early in a fire, when the energy flux was very small and the plume length very long, the entrainment was overpredicted. This resulted in the predicted interface falling more rapidly than was seen in experiments. Also the initial temperature was too low but the rate of rise too fast, whereas the asymptotic temperature was correct. The latter occurred when these early effects were no longer important.

The correct sequence of events is for a small fire to generate a plume which does not reach the ceiling or upper layer initially. The fire entrains enough cool gas to decrease the buoyancy to the point where the plume no longer rises[6]. When there is sufficient energy present in the plume, it

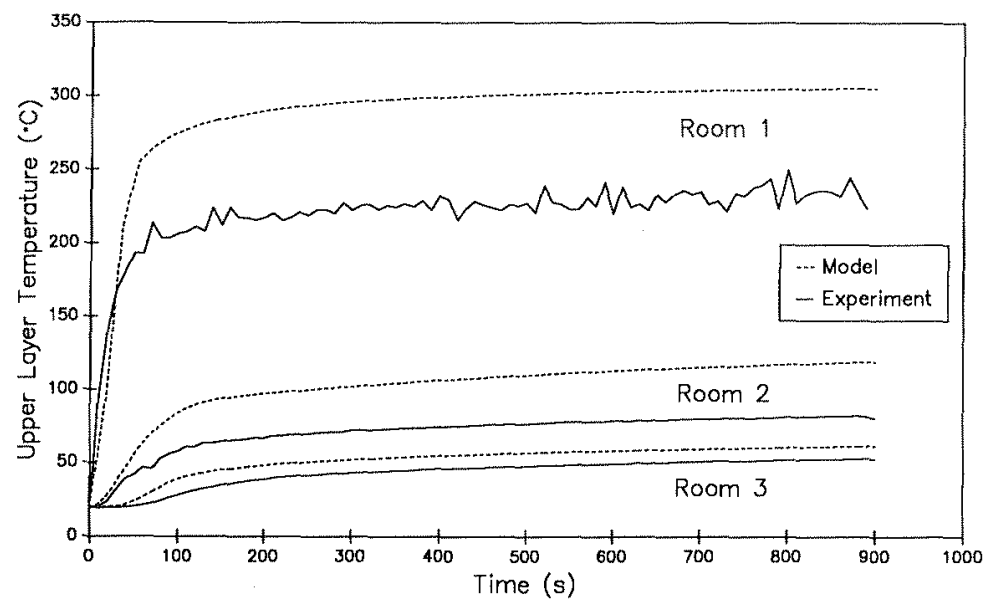

Figure 1: Comparison of Model Predictions and Experimental Measurements of Upper Layer Temperature in a Three Room Test Facility 


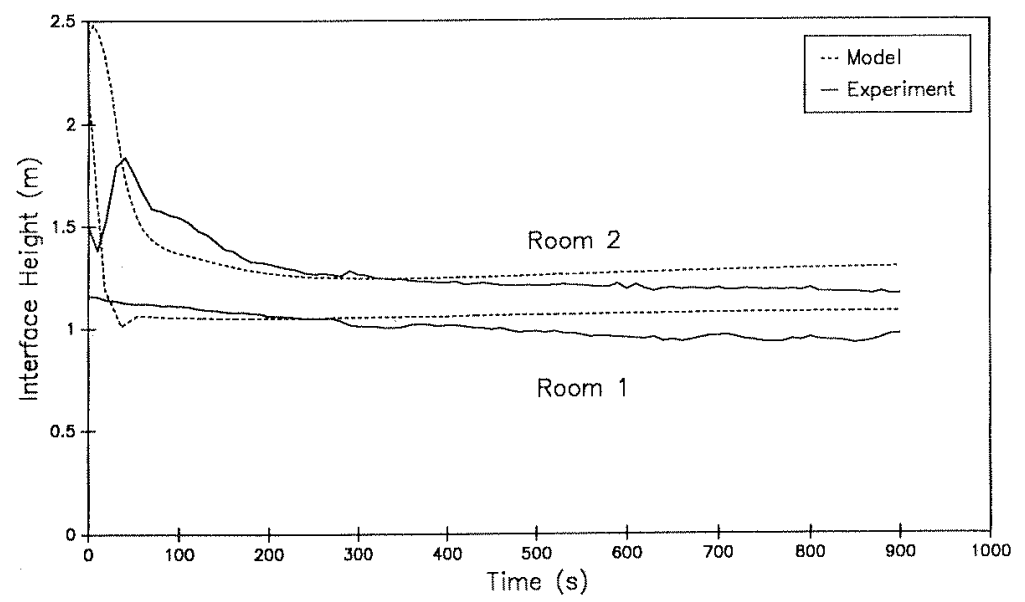

Figure 2: Comparison of Model Predictions and Experimental Measurements of Layer Interface Position in a Three Room Test Facility

will penetrate the upper layer. The effect is two-fold: first, the interface will take longer to fall and second, the rate of rise of the upper layer temperature will not be as great. To this end the following prescription has been incorporated: for a given size fire, a limit is placed on the amount of
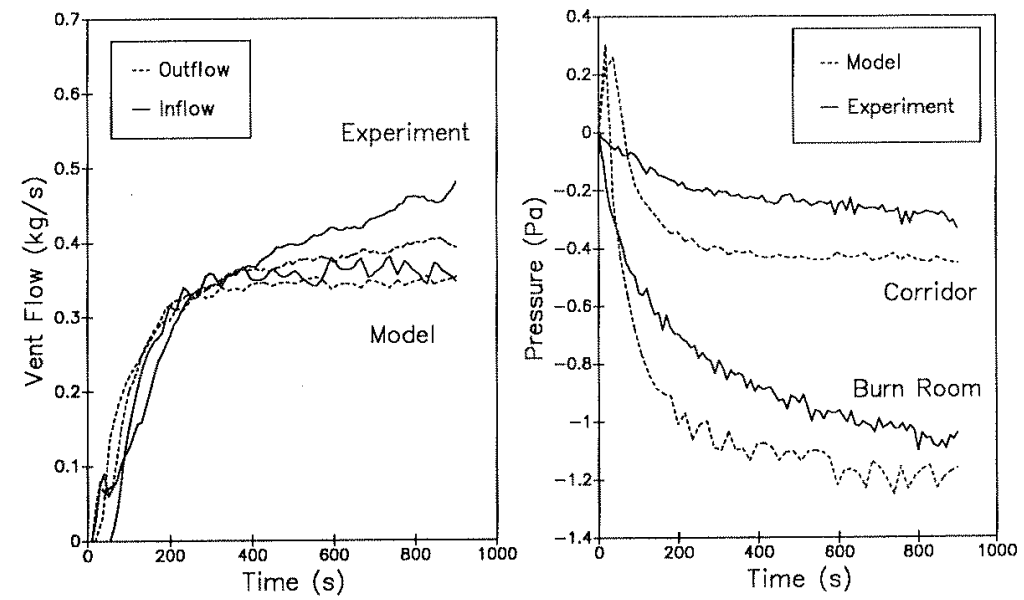

Figure 3: Comparison of Model Predictions and Experimental. Measurements of Flow in a Vent and Pressure in a Three Room Test Facility

mass which can be entrained, such that no more is entrained than would allow the plume to reach the layer interface. The result is that the interface falls at about the correct rate, although it starts a 1 ittle 
too soon, and the upper layer temperature is slightly overpredicted but after the initial phase, follows the experimental data very we11.

The opposite problem is encountered in the flow through vents. The entrainment is underestimated. The difficulty lies in the use of a plume model well beyond its nominal range of validity. The work of McCaffrey [7] really only applies to a circular plume. In the case of vent flow we encounter an extended flat plume similar to a waterfa11; however, there exists no reliable correlation for this configuration as yet. Thus we must presume to use the former correlation until a better plume model can be developed.

Many of the comparisons used for verification and validation of these types of predictive models do not include the peripheral a1gorithms, as much of this information is not of immediate interest. An example is the surface temperature of the walls of a building. However, the comparison is worthwhile for two reasons: first, eventually information such as wall temperatures can be coupled to models which consider the structural integrity of buildings; second, although the algorithms are checked for internal consistency, generally there is no overall check on the absolute validity of the emissivities, absorption coefficients, etc., which we use. Figure 1 includes such a comparison for the upper wall surface temperature in the middle of the corridor. Comparable information is not available for the burn room. The agreement is quite good.

The third comparison is of flow through vents. This is of great interest both from a theoretical standpoint and from the point of view of modeling hazard in a fire. Figure 3 shows a comparison of the flow between the corridor and the outside. Initially, the flow out of a room which contains a fire will exceed the inflow. During this period, expansion of the gases occurs due to heating, and the compartment is being filled from the top down. Once the interface reaches the soffit, then bidirectional flow occurs. As we approach an asymptotic limit of the experiment, the inflow and outflow should approximately match, with possible differences due to long term heating. From the point of view of the experiment, this is a very difficult measurement. In the data reduction, there is no attempt to allow for multiple neutral planes, whereas the model can do this quite easily. By comparison, the flow at the exit vent in the corridor shows inflow and outflow to be approximately equal and to agree quite well with theory[5].

A comparison between predicted and calculated pressures is also shown in figure 3. They agree very we11. The range is about one Pascal, starting with an initial bump of about one Pascal and decreasing to $1 / 2$ Pascal in the limit of long time. This latter agreement is very important since the prime motivation in making the refinements discussed here is to be able to track these very small pressure defects.

\section{CONCLUSIONS}

A transport model for fires in enclosures has been presented whose predictions of extensible quantities compares favorably with experimental measurements of these quantities. As with any theoretical model there are pieces which have been omitted and others which could be implemented more completely. Given the limitations, the model seems to do a credible job. As as can be seen by comparison with earlier calculations, the 
predictive capability is better in the area of small pressure differences, which is very important for future applications of this work.

\section{NOMENCLATURE}

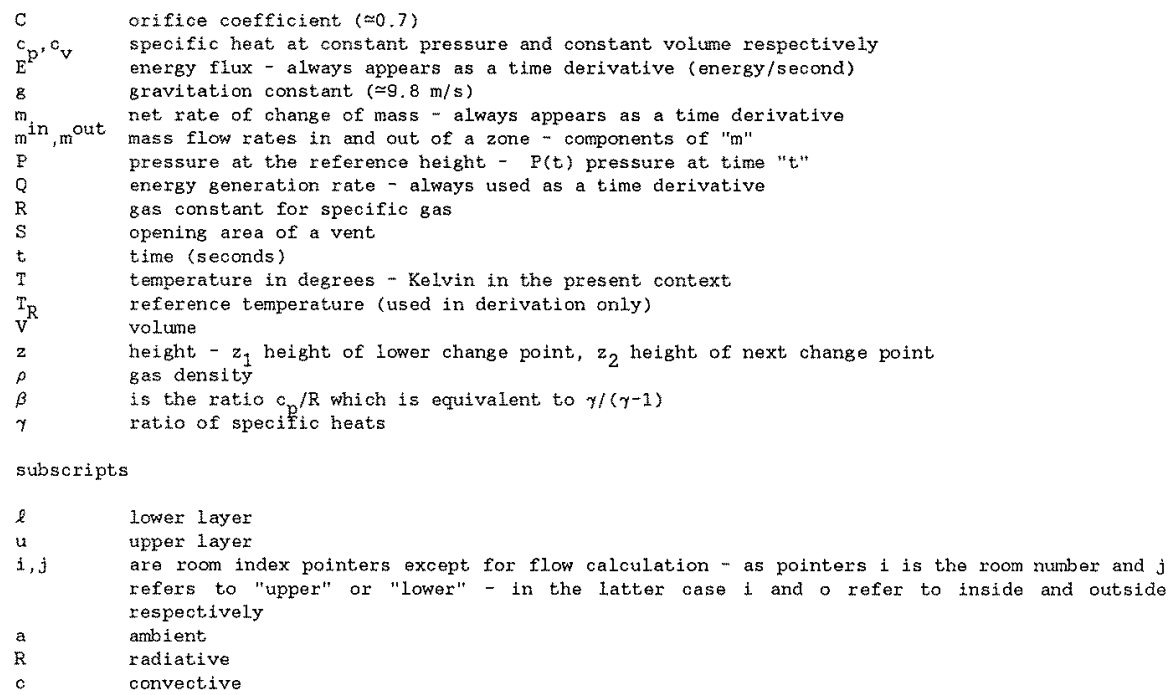

\section{BIBLIOGRAPHY}

[1] Jones, W.W., Refinement of a Model for Fire Growth and Smoke Transport, in review (1987).

[2] Quintiere, J.G., Steckler, K., and Corley, D., An Assessment of Fire Induced Flows in Compaxtments, Fire Science and Technology 4,1 (1984).

[3] Jones, W.W. and Bodart, X., Buoyancy Driven Flow as the Forcing Function of Smoke Transport Models, IR 86-3329, National Bureau of Standards (USA) (1986)

[4] Goldman, D, and Jaluria, Y., Effect of Buoyanoy on the Flow in Free and Wall Jets, ASME Winter Amnual Meeting, Miami (1985).

[5] Peacock, R.D., Davis, S., Lee, B.T., An Experimental Data Set for Verification of Room Fire Models, National Bureau of Standards (USA) Report NBSIR 87-xxxx, in review (1986).

16] Chen, C.J., and Rodi, W., Vertical Turbulent Buoyant Jets, Pergonon Press, page 61ff, New York (1980).

[7] McCaffrey, B.J., Momentum Implications for Buoyant Diffusion Flames, Combustion and Flame 52 $149(1983)$. 